\title{
Artificial Intelligence Robotically Assisted Brain Surgery
}

\author{
A. Jerwin Prabu ${ }^{1}$, J. Narmadha ${ }^{2}$, K. Jeyaprakash ${ }^{3}$ \\ ${ }^{I}$ ECE Department., Karpagam University, Coimbatore, India. \\ ${ }^{2}$ Assistant Professor, ECE Dept, SNS College of Technology, Coimbatore, India. \\ ${ }^{3}$ Assistant Professor, Mechanical Dept., SNS College of Technology, Coimbatore, India.
}

\begin{abstract}
Artificial Intelligence is both the intelligence of machine, electronics and the branch of Computer which aim to create it. AI has produced many significant and impressive products even at this early stage in its development. Although no one can predict the future in details, it is clear that computers with human-level intelligence would have a huge impact on our everyday lives and on the future course of civilization. Robot simply acts as an active agent whose environment is the physical world the active part rules out rocks, the artificial part rules out pure software agents or soft bots, whose environment consists of computer, file systems, database and networks. In this paper, the concepts of surgical robots, how robotically assisted brain surgery performed and their benefits are discussed. The first generations of surgical robots are already being installed in a number of operating rooms around the world. Compared with traditional surgery, the Robotically-assisted brain surgery is very useful.
\end{abstract}

Keywords: - Artificial Intelligence, Blood Brain Barrier, Computed Tomography, Gold Plated Electrode, Magnetic Resonance Imaging, Neural Networks, Diathermy.

\section{INTRODUCTION}

A know ledge of the local solar-radiation is essential for the proper design of building energy systems, Robotically assisted surgery also is called computer assisted surgery. This surgery is termed as technological development. Using the sensors it can identify the tumor cells. There are several disposable sensors where the sensor is located externally from the body although body fluids come in contact with it. Selecting a sensor can be simple if the application and parameters that need to be monitored are clearly understood. Today, many robot enhancements are being researched and developed. So, these instruments are using in this surgery. Only then the robotically assisted brain surgery will perform very well. Using this technology it will be very useful for many people and also to our next generation. Artificial Intelligence is the study of how to make computers do things which, at the moment, people do better. The art of creating machines that performs functions that require intelligence when performed by people. A re programmable, multi functional manipulator designed to move material, parts, or specialized devices through various tools, or specialized devices through various programmed motions for the performance of a programmed motion for the performance of a variety of tasks. A robot may not injure a human being, or through in action, allow a human being to come to harm. A robot must obey orders given it by human beings, except where such orders would conflict beings, with the First Law. A robot must protect its own existence as long as such protection does not conflict with the First or Second Law. An agent is anything that can be viewed as perceiving its environment through sensors and acting upon that environment through effectors. Human agent has eyes, ears and other organs for sensors, and hands, legs, mouth and other body parts for effectors. A robotic agent substitutes cameras and infrared range finders for the sensors and various motors for the effectors. There are so much of problems in artificial intelligence. They are deduction, reasoning, problem solving, Knowledge Representation, Planning, Learning, Natural language processing, Motion and manipulation, Perception, Social intelligence, Creativity, General intelligence. Many of the problems above are to be solved. Search algorithm, logic programming, Probabilistic methods for uncertain reasoning, Classifiers and statistical learning methods and also neural networks are important.

\section{ARTIFICIAL NEURAL NETWORKS}

A neural network is an interconnected group of nodes, akin to the vast network of neurons in the human brain. Neural networks are applied to the problem of learning, using such techniques as Hebbian learning, Holographic associative memory and the relatively new field of Hierarchical Temporal Memory which simulates the architecture of the neo cortex. Artificial intelligence has been used in a wide range of fields including medical diagnosis, stock trading, robot control, law, scientific discovery and toys. Frequently, when a technique reaches mainstream use it is no longer considered artificial intelligence, sometimes described as the AI effect. It may also become integrated into artificial life. 


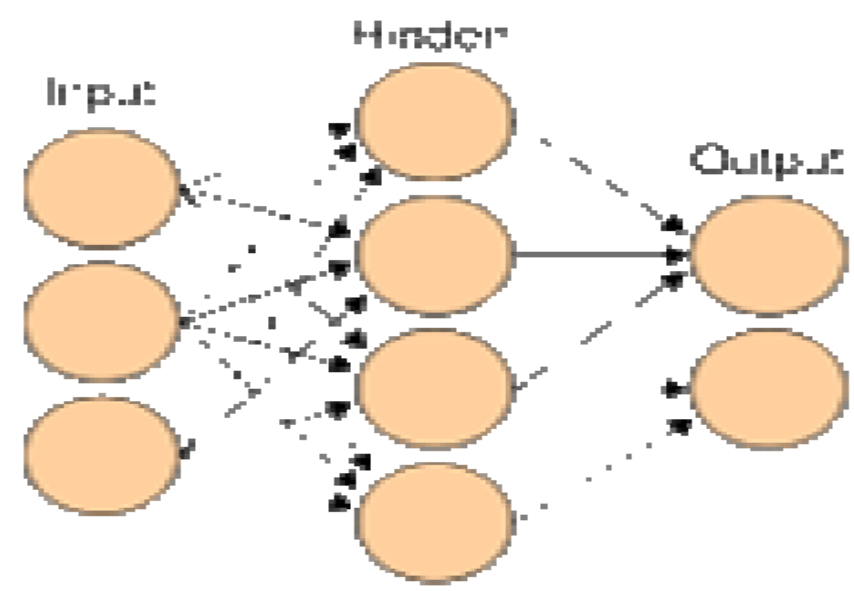

Fig. 1 Artificial Neural Network

\section{ROBOTS IN SURGERY AND TOOLS}

Surgical robots will be approved for use in operating rooms. We will also take a look at the advantages and benefits that robotic surgery will have over conventional surgical methods. Three main steps can be pointed out in a general robotic surgery intervention data acquisition and subsequent planning, intra operative assistance, and post-operative patient control. In the pre-operative phase, a patient dependent model of the rigid (e.g. bones), and de-formable (e.g. the brain, heart) anatomical entities involved in the surgical act have to be built. For this, several medical imagery techniques (MRI, Scanner, CT Scanner Ultrasonic's etc.) are used, where the anatomical structures are detected, located and modeled. In the same time, the mechanical model of the robotic system is fused in an overall geometric model. This will be used to describe and simulate the different potential problems that may occur during the intervention. The first generations of surgical robots are already being installed in a number of operating rooms around the world. These aren't true autonomous robots that can perform surgical tasks on their own, but they are lending a mechanical helping hand to surgeons. These machines still require a human surgeon to operate them and input instructions. Remote control and voice activation are the methods by which these surgical robots are controlled.

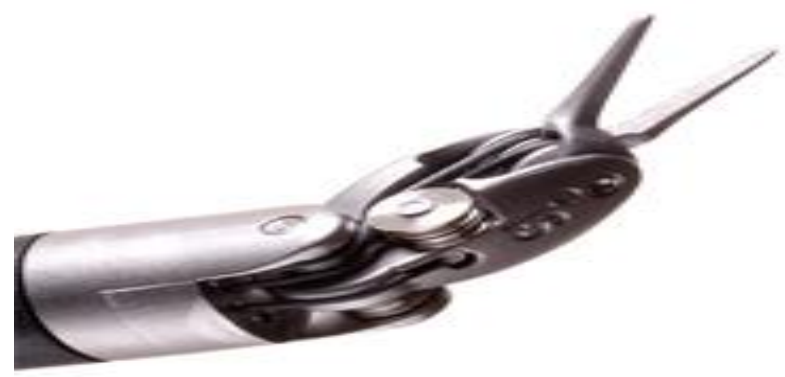

Fig. 2 Robotic Surgeon Tool

Robotics is being introduced to medicine because they allow for unprecedented control and precision of surgical instruments in minimally invasive procedures. So far, these machines have been used to position a CT scan, perform brain surgery and correctly identified and using the main brain surgery instrument called for Diathermy. It is used to remove the tumor with the help of robot. It is commonly used for muscle relaxation. It is also a method of heating tissue electromagnetically or ultrasonically for therapeutic purposes in medicine. According to one manufacturer, robotic devices could be used in more than 3.5 million medical procedures per year in the United States alone. The given figure represented as a diathermy tool. This is also called as wireless transmission magnetron oscillator. Using this type of oscillator, the heat will produced after that the unwanted cells are destroyed. We are using an automobile solenoid why because it will give constant current, voltage and adjustable frequency level. 

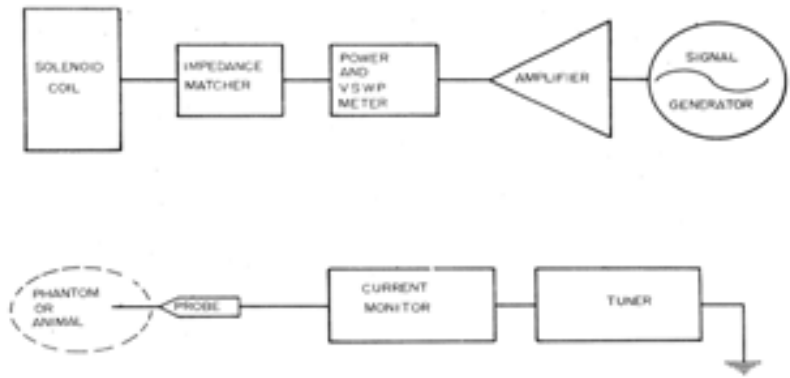

Fig. 3 Diathermy Block Diagram

Diathermy is used in physical therapy and occupational therapy to deliver moderate heat directly to pathologic lesions in the deeper tissues of the body. The ultimate goal of the robotic surgery field is to design a robot that can be used to perform brain tumor surgery. There are three surgical robots that have been recently developed but only for heart surgery.

- Da Vinci Surgical System

- ZEUS Robotic Surgical System

- AESOP Robotic System

\section{ROBOTIC SURGICAL SYSTEM USING SENSOR}

The computer-enhanced robotic system consists of three components, including a three-dimensional view of the surgical field, including depth of field, magnification and high resolution. This picture represents the gold plated cup shaped electrodes. This electrode is used to identify the tumor, which is one type of sensor.

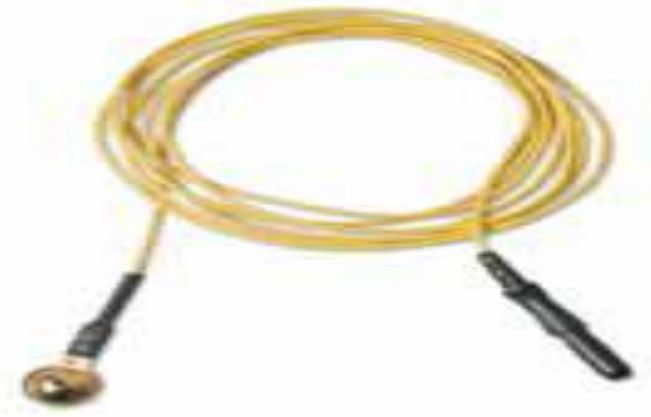

Fig. 4 Gold Platted Cup Shapely Electrode

Next we use the wireless transmission magnetron oscillator. Using this type of oscillator, heat is produced and it is used to remove the tumor. It allows an extensive range of motion and more precision. Master controls that allow the surgeon to manipulate the instruments, translating the surgeon's natural hand and wrist movements into corresponding, precise and scaled. That has been approved by the FDA for use in performing many surgical procedures. A major obstacle in tele-surgery has been the time delay between the doctors moving his or her hands to the robotic arms responding to those movements. Currently, the doctor must be in the room with the patient for robotic systems to react instantly to the doctor's hand movements or the doctors are outside in the room. First the skull is opened and after that the robot performs the surgery. It could lower the cost of health care. In addition to cost efficiency, robotic surgery has several other advantages over conventional surgery, including enhanced precision and reduced trauma to the patient.

\section{COMMON BRAIN TUMOR AND SURGICAL SYSTEMS}

\subsection{Symptoms}

- In the person's mental function, Headaches, Weakness in one part of the body

- Occur during sleep, Change in alertness, Changes in taste, smell

- Memory loss, Eye problems, Difficulty in writing and reading

5.2 Diagnosis The majority of the brain is separated from the blood by the blood-brain barrier (BBB) which exerts a restrictive control as to which substances are allowed to pass. Therefore many tracers that reach tumors in the body very easily would only reach brain tumors once there is a disruption of the BBB. Therefore the disruption of the BBB (blood-brain-barrier), which can be detected by a MRI and CT, is regarded as the main diagnostic indicator for malignant gliomas, meningiomas, and brain. 
5.3 Anaplasia Dedifferentiation: loss of differentiation of cells and of their orientation to one another and blood vessels is a characteristic of anaplastic tumor tissue metastases.

5.4 Radiation therapy The goal of radiation therapy is to selectively kill tumor cells while leaving normal brain tissue unharmed. In standard external beam radiation therapy, multiple treatments of standarddose "fractions" of radiation are applied to the brain. This process is repeated for a total of 10 to 30 treatments, depending on the type of tumor.

5.5 Chemotherapy Patients undergoing chemotherapy are administered drugs designed to kill tumor cells. Although chemotherapy may improve overall survival in patients with the most malignant primary brain tumors. But these all surgeries are restricted why because we are using alpha, beta, gamma, and Ultra Violet rays. These magnetic and frequency ranges are measured in tera hertz. So these frequency and waves are not good for health.

\section{BASE WORK - DA VINCI HEART SURGERY}

Robotically-assisted heart surgery, also called closed-chest heart surgery, is a type of minimally invasive heart surgery performed by a cardiac surgeon. The surgeon uses a specially-designed computer console to control surgical instruments on thin robotic arms. Robotically-assisted surgery has changed the way certain heart operations are being performed. This technology allows surgeons to perform certain types of complex heart surgeries with smaller incisions and precise motion control, offering patients improved outcomes.

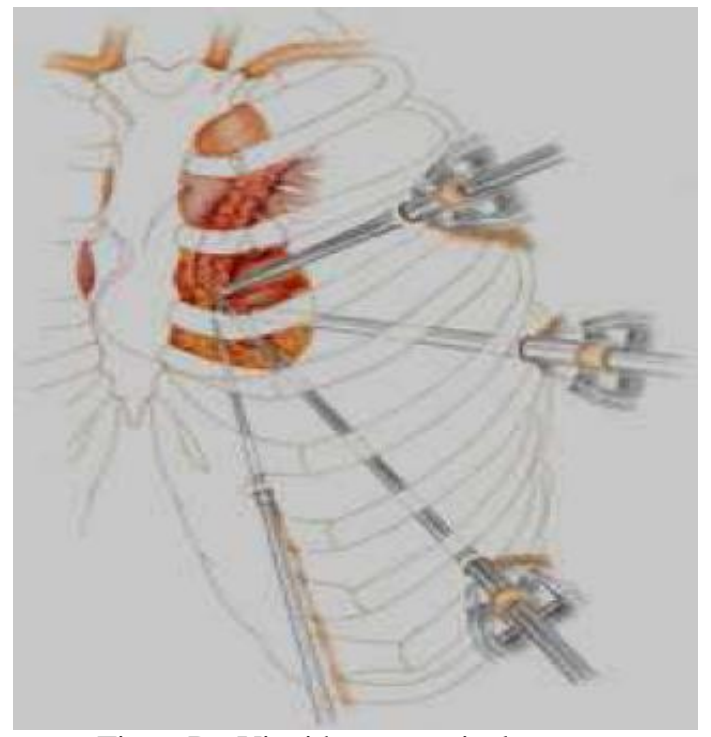

Fig. 6 Da Vinci heart surgical system

Three small incisions or "ports" are made in the spaces between the ribs. The surgical instruments (attached to the robotic arms), and one camera are placed through these ports. Motion sensors are attached to the robotic "wrist" so the surgeon can control the movement of the surgical instruments. The surgeon sits at a computer console and looks through two lenses (one for each eye) that display images from the specialized camera with two optical outputs.

\section{ROBOTICALLY ASSISTED BRAIN SURGERY AND ITS PERFORMANCE}

From the two optical outputs, the computer generates a clear, three-dimensional image of the surgical site for the surgeon to view. The system control pedals provide precise camera control, so the surgeon can instantly zoom in and out to change the surgical view. After that we will analyze the brain tumor range. 


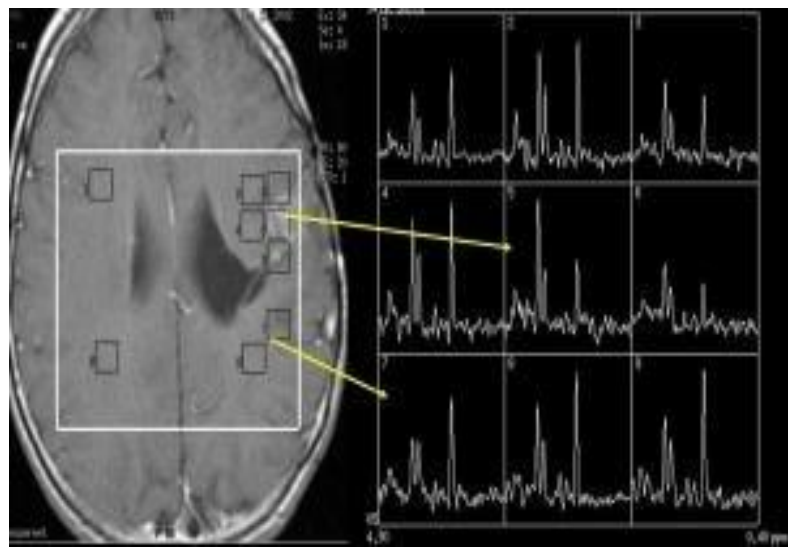

Fig. 7 Tumor Range Analyzing Report

7.1 Physical exam An exam of the body to check general signs of health, including checking for decease past illness and habits will also be taken. Analyze the brain signals using lab view software.

7.2 Neurological exam A series of questions and tests to check the brain.

7.3 Visual field exam An exam to check your field of vision. This test measures both central and peripheral vision.

7.4 Gene Testing A laboratory test in which a sample of blood or tissue is tested for changes in a genetic material that has been linked with a certain type of brain tumor.

7.5 CT, MRI, FMRI, PET Scans A neuroimaging procedure that makes a series of detailed picture of areas inside the body, taken from different angles. A neuroimaging procedure that uses a magnet, radio waves, and a computer to make a series of detailed picture of the brain and spinal cord. During an FMRI, the patient is asked to perform certain activities to help the neurosurgeons map the functional area of the brain before surgery takes place. A PET scan is a neuroimaging technique used to find malignant tumor cells. It can be used to analyze the fluid in the spinal cord. Measuring whether certain cancers have spread to the brain. These tests are taken and determine the patient mental function and organ function, including a CT scan and, in most MRI. An Electro encephala gram and/or a computed PET scan also may be required to provide more information about his medical condition. The surgeon will review the results of these diagnostic tests to determine if robotically assisted BRAIN surgery is the right treatment approach for him. The type of treatment recommended for his condition will depend on several factors, including the type and severity of BRAIN TUMOR disease, his age, medical history and lifestyle.

\section{RESULTS AND DISCUSSIONS}

Compared with traditional surgery, the benefits of robotically-assisted surgery we don't want to open skull, or smaller incisions with minimal scarring, less pain. Decreased use of pain medications, Less bleeding, Decreased risk of infection Shorter recovery and quicker return to daily and professional activities: The patient can resume normal activities and work as soon as he or she feels up to it; there are no specific activity restrictions after robotically-assisted surgery. In today's operating rooms, you'll find two or three surgeons, an anesthesiologist and several nurses, all needed for even the simplest of surgeries. Most surgeries require nearly a dozen people in the room. As with all automation, surgical robots will eventually eliminate the need for some of those personnel. Taking a glimpse into the future, surgery may require only one surgeon, an anesthesiologist and one or two nurses. In this nearly empty operating room, the doctor will sit at a computer console, either in or outside the operating room, using the surgical robot to accomplish what it once took a crowd of people to perform. The use of a computer console to perform operations from a distance opens up the idea of tele-surgery, which would involve a doctor performing delicate surgery miles away from the patient. If the doctor doesn't have to stand over the patient to perform the surgery, and can remotely control the robotic arms at a computer station a few feet from the patient, the next step would be performing surgery from locations that are even farther away. If it were possible to use the computer console to move the robotic arms in real-time, then it would be possible for a doctor in California to operate on a patient in New York. Finally we have to analyze the tumor range and produce signal wave forms using lab view software and sensors. 


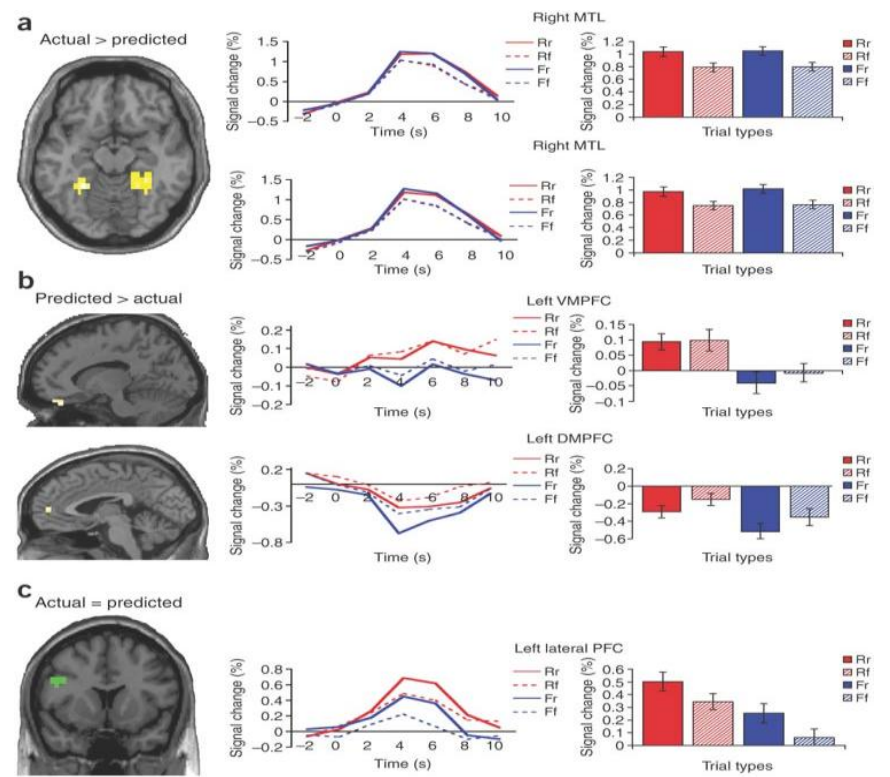

Fig. 8 Reference Output Flow Graph

\section{CONCLUSION}

The current development within the fields of surgical robots, robotically assisted heart surgery described here is a step in right direction. The robotic surgeon as da Vinci Surgical System and ZEUS Robotic Surgical System play an important role in heart surgery system. The surgeon is always in control during the brain surgery, there is no chance that the robotic arms will move on their own. That is very safe. The robotically assisted brain surgery plays an important role in the medical field. In future, improvement in the developments in robotic surgery will be seen.

\section{REFERENCES}

[1] Natarajan P, Krishnan's, Natasha Sandeep Kenkre, Shraiya Nancy, Bhuvanesh Pratap Singh, "Tumor Detection using threshold operation in MRI Brain Images", IEEE International Conference on Computational Intelligence and Computing Research, 2012.

[2] Prachi Gadpayleand, P.S. Mahajani, "Detection and Classification of Brain Tumor in MRI Images International Journal of Emerging Trends in Electrical and Electronics, IJETEE - ISSN: 2320-9569, Vol. 5, Issue. 1, July-2013.

[3] Mohd Fauzi Othman, Mohd Ariffanan, Mohd Basri, "Probabilistic Neural Network for Brain Tumor Classification", IEEE International Conference on Intelligent Systems, Modeling and Simulation, 2011.

[4] Stuart J.Russell and Peter Norvig, “Artificial Intelligence a Modern Approach”, Pearson Education Series in Artificial Intelligence (2003)., 91(1a):106-15. (1985).

[5] T.J. Cartwright, Dr.Monisha E.Michlen, Dr.Juan Crestanello, sowmiya Createectomy, roboticwhipple surgery, Procedures University at Chicago, 1999-2002

[6] Reza S. Dilmaghani, Hossein Bobarshad, M Ghavami, Sabrieh Choobkar, and Charles Wolfe "Wireless Sensor Networks for Monitoring Physiological Signals of Multiple Patients" IEEE transactions on Biomedical circuits and systems, vol. 5, no. 4, august 2011 online at http://ieeexplore.ieee.org.

[7] A b. sirisha, t.sraddha, $k$. vijayanand "Real-time multi-patient monitoring system using arm and wireless Sensor network" International Journal of Communication Network Security, ISSN: 2231 - 1882 , Volume-2, Issue-2, 2013

[8] H Juney M George1, Venugopal G "Design of an embedded platform for patient monitoring applications using $\mu \mathrm{c}$ os-ii” International Journal of Advanced Research in Electrical, Electronics and Instrumentation Engineering Vol. 2, Special Issue 1, December 2013 online at www.ijareeie.com

[9] Edward Fredkin, Ranjitha, suba, Charles T.Rubin AI is the next Stage in evaluation, Samuel Butler's Darwin Among the machines book,[1863][1998]-317.32.179

[10] Yadav Satyendra Satyanarayan, Yadav Raghvendra Satyanarayan, Deep H. Desai "Intelligent Wireless Emergency Alert System for Patient Monitoring using AT89S52 Microcontroller" International Journal of Advanced Research in Electrical, Electronics and Instrumentation Engineering Vol. 2, Issue4, April 2013 online at www.ijareeie.com 